\title{
Robust automatic segregation of harmonics in electric drives by means of the Mahalanobis distance
}

\author{
Omer Ikram Ul Haq ${ }^{1}$, Luca Peretti ${ }^{1}$ \\ ${ }^{1}$ Dept. of Electrical Systems, ABB Corporate Research, Västerås, Sweden, [omer.ikramulhaq],[luca.peretti]@se.abb.com
}

\begin{abstract}
This paper discusses the use of the Mahalanobis distance as a solution for a robust and automatic segregation of harmonics in the signals measured in electric drives, as for example the phase currents or the mechanical speed. The Mahalanobis distance is used during real-time operation to clean the results of an on-line frequency analysis, reducing the uncertainties related to frequency resolution, spectral leakage and background noise. At the same time, it is a powerful tool to classify the known harmonics from the unknown ones, thus providing the drive with valuable information that can be used for further decisional control actions. The overall theoretical background is reviewed as well as its implementation in an operational drive system. Experimental results are provided, proving the validity of the concept.
\end{abstract}

Keywords-Electric drives; diagnostics; harmonics; frequency analysis; Mahalanobis distance

\section{INTRODUCTION}

The computational capabilities of electric drive systems are increasing as a consequence of the development of microprocessors and FPGA used in modern control boards. This allows for the implementation of control algorithms and other software features which can potentially go beyond the conventional control tasks of speed and torque regulation of an electric machine. In this scenario, one of the most interesting trends is represented by monitoring and diagnostic software features implemented directly in the drive control board, leveraging on the existing hardware and the existing sensors. These solutions contrast with the conventional approach of using additional, external equipment to perform diagnostic, and aim at a further reduction of the installation costs without losing diagnostic performances.

The scientific field of diagnostics for drive systems is very prolific and several studies have been published in order to relate the presence of footprints in measured signals to incipient faults in the system. In particular, this work is interested in those harmonics that could reveal unexpected malfunctioning of the electric machine, the drive or the mechanical load.

Some literature examples are discussed here below. It is known that some harmonics are generated by the electric machine itself, as for example the cogging torque which appear as a multiple of the sixth harmonic of the electric frequency [1]. While cogging torque is not an indication of a failure, its interaction with the mechanical system could excite mechanical resonances, resulting in high vibrations that could potentially harm the drive train [1]. It has been also found that rotor faults in synchronous machines reflect in a number of frequency components appearing in the stator currents [2]. The frequency converter can also contribute to the generation of harmonics, as shown for example in [3] where the poor compensation of the IGBT dead times lead to sixth harmonics of the electric frequency in the dq currents and the torque.

Mechanical load unbalances and shaft misalignments may also introduce measurable oscillations in the stator currents [4]. Moreover, specific energy conversion applications may introduce oscillations, as the case of wind shear and tower shadow effects in wind turbines which account for a third harmonic of the rotating frequency [5].

In principle, most of these harmonics are detectable by measuring the phase currents and/or the mechanical speed, and by performing a spectral analysis of their time-domain waveforms. Several algorithms for frequency-based and timebased spectral analysis are available from the literature, from the conventional fast Fourier Transform (FFT) [6], to the sparse FFT [7], as well as linear prediction analysis [8]. These algorithms are not discussed in this paper, although they represent a very important part of a diagnostic system.

The focus of this paper, instead, is directed to a classification algorithm that can systematically segregate the results of a spectral analysis, in order to recognise known harmonics without posing any initial assumptions on the frequencies that should be found in the spectrum itself. This approach is fuelled by the consideration that, while diagnostic algorithms that search for the appearance of specific frequencies are certainly possible, typical electric drive applications possess little prior knowledge on the electric machine that will be operated. Thus it is somewhat difficult to focus on the search of some frequencies, while leaving others out of the analysis. In other words, it seems that a generalised approach for a robust and possibly automatic detection and classification of harmonics is missing.

The proposed classification algorithm is based on the concept of Mahalanobis distance, a fairly mature concept with very interesting mathematical and statistical implications [9]. The paper is organised as follows. Sect. II presents the theory of the Mahalanobis distance. Sect. III discusses how the Mahalanobis distance could be proactively used in electric drive applications, and more in particular for the classification of harmonics in monitoring and diagnostic systems. Sect. IV describes the steps required to implement the classification algorithm in an electric drive control board, while Sect. V shows a series of experimental results that validate the method. Some conclusive remarks will end the paper. 


\section{THE MAHALANOBIS DISTANCE}

The empirical values obtained from a series of $N$ experimental observations of a phenomena can be mathematically described by a random vector $\boldsymbol{x}$ of dimension $n$, where each element $\boldsymbol{x}_{i}, i \in[1, \ldots, n]$, is a scalar random variable containing $N$ different observed values. When the expected value of each scalar random variable $\mu_{i}=E\left[x_{i}\right]$ is calculated, a vector $\boldsymbol{\mu}$ is obtained:

$$
\boldsymbol{\mu}=\left[\mu_{1}, \mu_{2}, \ldots, \mu_{n}\right]^{T}
$$

In this case, considering that the observations are equally likely in statistical terms, the expected values coincide with the mean values of the scalar random variables $\boldsymbol{x}_{i}, i \in[1, \ldots, n]$, and therefore the vector $\boldsymbol{\mu}$ contains the mean value of each dimension of the random vector $\boldsymbol{x}$ across the whole set of observations.

When the scalar random variables possess a finite variance, defined as the expectation of the squared deviation of a random variable from its mean value, a covariance matrix $\boldsymbol{C}$ can also be defined. Its elements $C_{i j}$ are calculated as the covariance $\operatorname{cov}\left(\boldsymbol{x}_{i}\right.$, $\left.\boldsymbol{x}_{j}\right)$ of the scalar random variables $\boldsymbol{x}_{i}$ and $\boldsymbol{x}_{j}, i \in[1, . ., n]$. The covariance is an indication of the joint variability of two scalar random variables, and according to its definition it is calculated by the following formula:

$$
C_{i j}=\operatorname{cov}\left(\boldsymbol{x}_{i}, \boldsymbol{x}_{j}\right)=E\left[\left(\boldsymbol{x}_{i}-\mu_{i}\right)\left(\boldsymbol{x}_{j}-\mu_{j}\right)\right]
$$

Considering again $N$ equally likely experimental observations, the covariance matrix becomes:

$$
C_{i j}=\frac{1}{N-1} \sum_{k=1}^{N}\left(x_{i k}-\mu_{i}\right)\left(x_{j k}-\mu_{j}\right)
$$

where $x_{i k}$ and $x_{j k}$ are the $k^{\text {th }}$ observations $(k \in[1, \ldots, N])$ of the scalar random variables $x_{i}$ and $x_{j}$, respectively. The covariance matrix $\boldsymbol{C}$ is a generalisation of the notion of variance to multiple dimensions, where a single scalar value is not able to characterise the variation of a collection of points in a $n$ dimensional space.

Given a mean vector $\boldsymbol{\mu}$ and a covariance matrix $\boldsymbol{C}$ describing a set of observations, a new observation $\boldsymbol{x}_{o}=\left[x_{o 1}, x_{o 2}\right.$, ..., $\left.x_{o n}\right]$ can be related to the original set of observations by the definition of a distance which takes into account the characteristics of the random vector $\boldsymbol{x}$. This distance, known as Mahalanobis distance and firstly defined in [9], is calculated as follows:

$$
d_{m}=\sqrt{\left(\boldsymbol{x}_{o}-\boldsymbol{\mu}\right)^{T} C^{-1}\left(\boldsymbol{x}_{o}-\boldsymbol{\mu}\right)}
$$

where $d_{m}$ is the Mahalanobis distance value. The Mahalanobis distance is considered a generalised version of the Euclidean distance, which is obtained from (4) when the matrix $\boldsymbol{C}$ is the identity matrix. Intuitively, the Mahalanobis distance preserves not only the information of the average value of a set of observations, but also their trend, and this comes particularly useful when the relation between $n$ scalar random variables must be taken into account.

A simple example in a two-dimensional space clarifies the differences between the Euclidian and the Mahalanobis distance. Let first assume that a series of $N$ experimental observations have defined the trend line of the function $\boldsymbol{x}_{2}=6 \boldsymbol{x}_{1}$, and that the mean vector $\boldsymbol{\mu}$ and covariance matrix $\boldsymbol{C}$ have been calculated according to (1) and (3). The trend line and the mean value are reported in Fig. 1, with a dashed line and a + symbol, respectively.

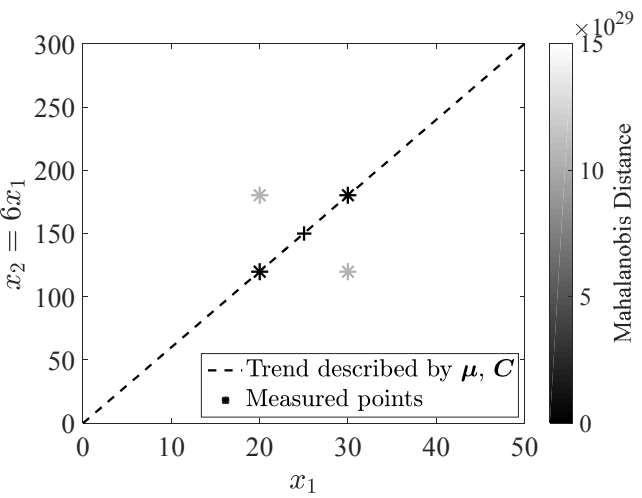

Fig. 1. Mahalanobis distance example.

Four new observations are taken and reported in the same Fig. 1 with four different $*$ symbols. Their color intensity is relative to their Mahalanobis distance to the trend line.

Two of the observations lie clearly on the trend line, while the other two lie at a certain distance to it. By calculating the Euclidian distance of the four observations with respect to the mean value indicated by the + symbol, one would notice that such distance is the same for all four cases. However, as it is visible on the scale reported on the right of Fig. 1, the Mahalanobis distance of the two observations that lie on the trend line is considerably smaller than the one calculated for the two observations that lie outside the trend line. It is therefore immediate that the Mahalanobis distance is a powerful tool that could be exploited for more sophisticated statistical data analysis. As described in the next Sect. 2, a keen use of the Mahalanobis distance for electric drive applications allows an increased robustness in the detection of harmonics in drive systems.

Before considering the application case, it is important to underline an important theoretical consideration on the calculation of the covariance matrix $\boldsymbol{C}$, which has an impact on the proposed use of the Mahalanobis distance. Assuming that the covariance matrix of the theoretical function $\boldsymbol{x}_{2}=f\left(\boldsymbol{x}_{1}\right)$ is sought, it is not sufficient to precisely apply the function $f$ to produce theoretical observations of $\boldsymbol{x}_{l}$ and $\boldsymbol{x}_{2}$. In the example of Fig. 1, this would mean producing theoretical observations of $\boldsymbol{x}_{1}$ and $\boldsymbol{x}_{2}$ such that $x_{2 k}=6 x_{l k}$, for $k \in[1, \ldots, N]$, and then use the theoretical observations to calculate the matrix $\boldsymbol{C}$. This will lead to a singular, non-invertible matrix $\boldsymbol{C}$, which cannot be used for the Mahalanobis distance calculation in (4). An 
invertible $\boldsymbol{C}$ is obtainable only when a random variability is present in the observations. Such variability can be well obtained by additive noise superimposed to the observations, or by allowing the observations to fall within a certain interval around the function $\boldsymbol{x}_{2}=f\left(\boldsymbol{x}_{1}\right)$. This consideration will come back in its full meaning in the following Sect. III.

\section{THE MAHALANOBIS DISTANCE FOR DRIVE DIAGNOSTICS}

The theoretical considerations of Sect. II on the Mahalanobis distance are now applied for an electric drive application. In particular, its properties are investigated for the case of a variable speed drive where the spectral content of measured or estimated signals (phase currents, DC bus voltage, and mechanical speed) is analysed and classified to possibly reveal incipient system failures. Within this context, the Mahalanobis distance finds immediate use in the classification of spectral lines for revealing known harmonics within the system.

In order to clarify the case, it is assumed that the control board of the frequency converter is able to execute the conventional feedback control for an electric machine (fieldoriented control, direct-torque control or similar alternatives) and, in parallel, an algorithm for the calculation of the spectrum of the measurable signals where the footprint of the incipient failure is sought. In the rest of the paper, the FFT will be mentioned as the spectral analysis tool, for easiness of description.

Assuming that the calculation of the spectrum is available at regular intervals, the analysis of the results could be focused to the search of specific frequencies representative of an incipient fault. As mentioned in Sect. I, the available literature in the field of diagnostics demonstrate that several frequencies could be related to unexpected malfunctioning in drive systems. However, a direct search of the frequencies with significant magnitude, without any attempt to classify the results with a systematic approach, seldom accounts for the natural inaccuracies of a digital analysis based on sampled signals with spectral leakage and superimposed noise, in a system where transient phenomena could occur at any time. In particular, three aspects may hamper the direct search:

- The frequency resolution of the spectral analysis, which in the case of the FFT is equal to the inverse of the analysed time window. A sought frequency may not be found if not a multiple of the frequency resolution.

- Even by using window functions (Hanning or BlackHarris, etc.) for spectral leakage reduction [10], a small amount of spectral leakage might remain. A classification algorithm based only on spectral line magnitude thresholds may find the sought frequency together with a series of side lobes of significant magnitude.

- Particularly for spectral lines with small magnitude, noise may play a role in recognising the presence of incipient failures. Again, classification algorithms purely based on a magnitude threshold detection may not be a suitable choice.
To improve the overall robustness of failure detection based on the spectral analysis of measured signals with respect to the issues of frequency resolution, spectral leakage and noise, this paper proposes to exploit the Mahalanobis distance to correlate significant frequencies in the spectrum with an available database of trend lines, each describing a harmonic relative to a known failure of the system.

The proposed method works as follows. By considering again the example of Fig. 1, the scalar random variable $\boldsymbol{x}_{l}$ is associated to the fundamental frequency of the signal under analysis. For example, $\boldsymbol{x}_{l}$ could be the mean value of the mechanical speed in the time window used by the FFT, which is related to the fundamental rotational frequency of the rotor. The fundamental current magnitude could also be used, as well as other signals. The scalar random variable $\boldsymbol{x}_{2}$, instead, is associated with a harmonic of interest in the system. For example, one may be interested in revealing third harmonics of the torque indicative of phenomena of wind shear and tower shadow in wind turbine drive trains [5], thus calculating the mean value $\boldsymbol{\mu}$ and the covariance matrix $\boldsymbol{C}$ of the function $\boldsymbol{x}_{2}=3 \boldsymbol{x}_{1}$. Several instances of $\boldsymbol{x}_{2}$, each with their own $\boldsymbol{\mu}$ and $\boldsymbol{C}$, could be considered to map several harmonics of interest in the system. Fig. 2 reports the case of the trend lines calculated for the $1^{\text {st }}, 2^{\text {nd }}, 5^{\text {th }}$ and $7^{\text {th }}$ harmonic of the analysed signal. The easiness of harmonic description through simple straight lines is noticeable.

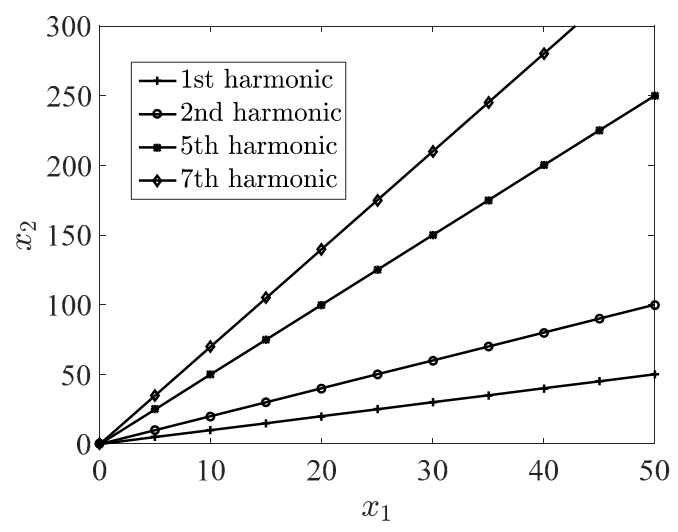

Fig. 2. Example of trend lines to describe multiple harmonics.

By assuming that several $\boldsymbol{\mu}$ and $\boldsymbol{C}$ are stored in the memory of the control board, the output of the spectral analysis performed by the FFT can be continuously checked against the available $\boldsymbol{\mu}$ 's and $\boldsymbol{C}$ 's, by calculating the Mahalanobis distance between the found significant frequencies and the trend lines. A low Mahalanobis distance is an indication of a known harmonics affecting the system, while a high Mahalanobis distance with respect to all trend lines represents an unknown phenomena, which may as well be a very important information for drive diagnostics. All in all, the Mahalanobis distance allows for a clear distinction between known and unknown harmonics affecting the system, becoming a suitable tool for harmonic segregation. In addition to this, the robustness of the method against frequency resolution, spectral leakage and noise is increased by adopting some implementation solutions that are described in the following Sect. IV. 


\section{THE IMPLEMENTATION IN AN ELECTRIC DRIVE}

\section{A. Overall description of the system}

A simplified schematic of the implemented system is shown in Fig. 3. It is assumed that the control board used to regulate the electric machine operation is able to execute a conventional electric machine feedback control based on a FOC or a DTC implementation, in parallel to a frequencydomain spectral analysis algorithm (the FFT, for example) and to the proposed harmonic classification algorithm based on the Mahalanobis distance.

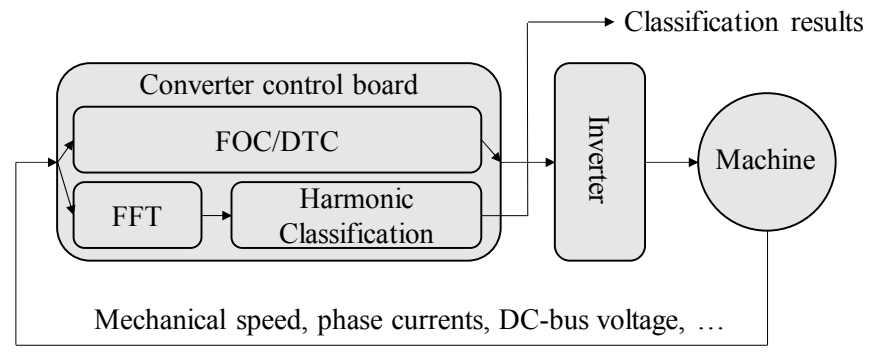

Fig. 3. Simplified schematic of the implemented system.

No further details will be given on the implementation of the spectral analysis algorithm, as it is not the goal of this paper. It is also assumed that the results of the harmonic classification are available outside the feedback control chain for monitoring purposes.

\section{B. Calculation and loading of the harmonic descriptions}

In Sect. III, it is mentioned that several $\boldsymbol{\mu}$ and $\boldsymbol{C}$ are stored in the control board memory, each describing a harmonic of interest. While the computational requirements to store $\boldsymbol{\mu}$ and $\boldsymbol{C}$ is minimal (in a two-dimensional space, $\boldsymbol{\mu}$ is a $2 \times 1$ vector and $\boldsymbol{C}$ is a 2x2 matrix), their offline calculation is not straightforward. Sect. II mentions that pure theoretical observations of the scalar random variables $\boldsymbol{x}_{1}$ and $\boldsymbol{x}_{2}$ are not sufficient for a correct calculation of $\boldsymbol{C}$, which would result in a non-invertible matrix. Some random variability must be introduced in order to obtain meaningful values for $\boldsymbol{C}$.

The need for random variability in theoretical observations relates very well to the purpose of the Mahalanobis distance to increase the robustness of the harmonic classification, by taking into account frequency resolution, spectral leakage and noise. Frequency resolution is a deterministic value obtained as the inverse of the time window used by the FFT: a time window of $T_{w} \mathrm{~s}$ allows for a frequency resolution of $\Delta f=1 / T_{w}$ $\mathrm{Hz}$. Therefore, each frequency with significant magnitude found in the spectrum by the FFT could possibly be related to any frequency in the interval $[f-\Delta f / 2, f+\Delta f / 2] \mathrm{Hz}$. Note that the meaning of this interval can be well extended to a complete trend line, providing a quantification of the accuracy related to the frequency resolution.

The effect of spectral leakage can be quantified in a similar manner. While looking for the location of frequencies with significant magnitude, the FFT may employ a Hanning window [10] to increase the probability of finding precise spectral lines. Although windowing the signal leads to considerably reduced spectral leakage, it is still possible that some side lobes around the main frequency could show a significant magnitude, and therefore they may be considered as significant frequencies as well. However, the side lobes are certainly an unwanted phenomena that should be cleared from the results of the analysis. The Mahalanobis distance allows for it, as long as a certain interval of frequencies around the trend line is still considered part of the trend line. In other words, given a function $\boldsymbol{x}_{2}=f\left(\boldsymbol{x}_{1}\right)$ describing a trend line, the frequencies allowed to belong to the trend line could be located in the interval $\left[f\left(\boldsymbol{x}_{l}\right)-k \Delta f, f\left(\boldsymbol{x}_{l}\right)+k \Delta f\right]$, with $k$ being a positive integer $(1,2, \ldots)$ whose value depends on the characteristics of the window function used by the FFT. Note that the selection of $k=1$ already incorporates the effects of the frequency resolution accuracy.

The effect of noise is the least quantifiable, and somehow relates to the quality and integrity of the signals in the drive system installations. No general indications are given here on how to incorporate the effect of noise. However, an approach similar to the one applied for the spectral leakage, allowing for a certain interval of frequencies to belong to the same trend line, seems to be a viable solution.

Looking back to the need of random variability in theoretical observations, and based on the above reported considerations, it is possible to define a certain interval around a trend line and randomly generate observations within the same interval, exclusively for the purpose of calculating $\boldsymbol{\mu}$ and $\boldsymbol{C}$. An example of a $6^{\text {th }}$-harmonic trend line description, with its interval accounting for frequency resolution, spectral leakage and noise, is reported in Fig. 4. In this example, considering a frequency resolution of $1 \mathrm{~Hz}$, the interval was selected as $\left[6 \boldsymbol{x}_{1^{-}}\right.$ $\left.40,6 \boldsymbol{x}_{1}+40\right] \mathrm{Hz}$ only for visualisation purposes. The theoretical observations with random variability used to calculate the values of $\boldsymbol{\mu}$ and $\boldsymbol{C}$ for the $6^{\text {th }}$-harmonic are reported as well.

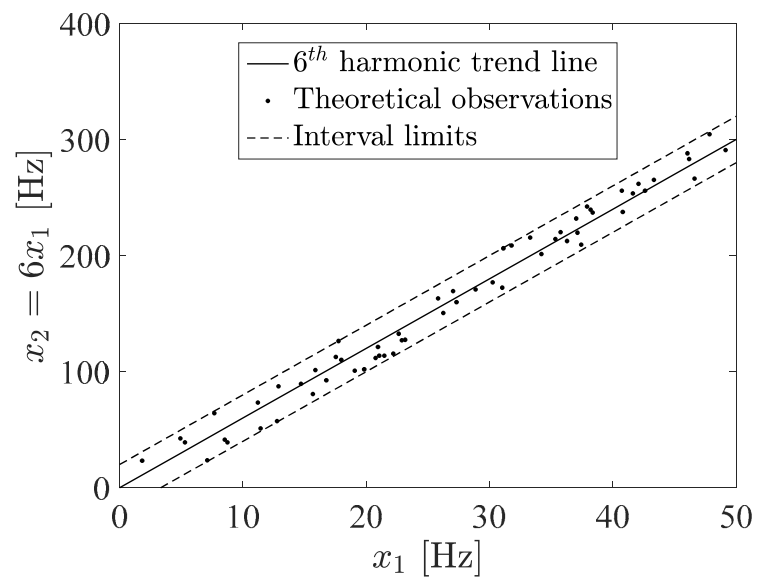

Fig. 4. Example of theoretical observations with random variability and interval limits to calculate $\boldsymbol{\mu}$ and $\boldsymbol{C}$ of a $6^{\text {th }}$-harmonic trend line.

\section{Mode of operation in real time}

Once the trend lines are saved into the memory of the control board, the classification algorithm is ready to operate in real time. A simplified schematic of the algorithm operation is shown in Fig. 5. 


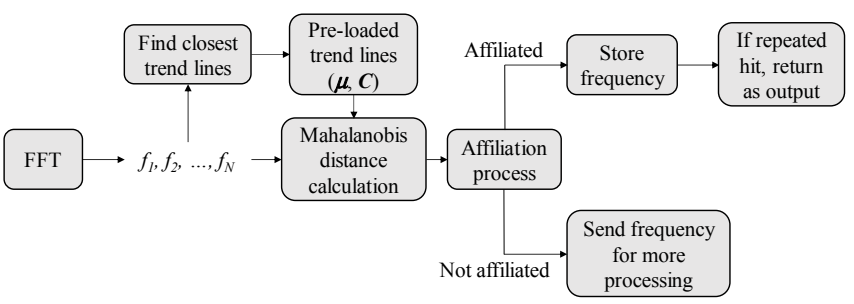

Fig. 5. Simplified schematic of the operation of the classification algorithm.

The FFT generates output frequencies at regular intervals dictated by its own time window, and a simple threshold analysis could be just used to reduce the operation of the classification algorithm to the most significant frequencies with relevant magnitude. Each of the significant frequencies coming as an output of the FFT (and their total number is not necessarily constant, therefore the classification algorithm must be execute for each of them) is used for two purposes:

- Identify the closest two trend lines by means of a simple check of the incoming frequency value with respect to the theoretical value of the trend lines. This is easily obtained by multiplying the slope of the trend line by the fundamental signal value representing $\boldsymbol{x}_{l}$. This first step is indeed not strictly necessary, but it helps in reducing the time execution of the classification algorithm.

- Calculate the Mahalanobis distance between the found frequency and the identified trend lines pre-stored in the memory.

Based on the value of the Mahalanobis distance (see more details in Sect. IV-D), the incoming frequency could be either affiliated to one harmonic or not affiliated to any harmonics described by the pre-stored trend lines. It is important to underline that the affiliation of a frequency does not automatically trigger the system to notify the existence of a known harmonic. The classification algorithm is configured to notify the existence of a known harmonic only if a significant frequency has been affiliated to it for most of some consecutive FFT time windows. For example, the existence of a harmonic might be notified only if the affiliation occurs for 6 times over 10 consecutive FFTs.

It is also worth to underline once more the capabilities of the classification algorithm in presence of spectral leakage. Assuming that the FFT returns significant magnitude for five very close spectral lines, the classification algorithm might be able to affiliate all of them to the very same harmonic, provided that the interval to which the frequencies are considered to belong to the same trend line has been correctly set. This would return as output just one harmonic hit, instead of five separate frequencies.

The missing affiliation to any of the pre-loaded trend lines is an indication that a significant frequency of unknown nature might be affecting the system. This information is send forward to the user for more data processing, which is deemed to be executed outside the control board.

\section{Low/high threshold values for the Mahalanobis distance}

Sect. IV-C describes that the affiliation to a certain trend line is evaluated by considering the value of the Mahalanobis distance of the incoming frequency with respect to the trend line. Thus, a threshold must exist for the discrimination of the affiliation.

Indeed, the frequency intervals which have been used to generate $\boldsymbol{\mu}$ and $\boldsymbol{C}$ for each trend line are also useful to determine the thresholds of the Mahalanobis distance. Fig. 6 shows a three-dimensional graph where the trend line and the interval limits shown in Fig. 4 are superimposed to the values of the Mahalanobis distance calculated for several possible theoretical observations.

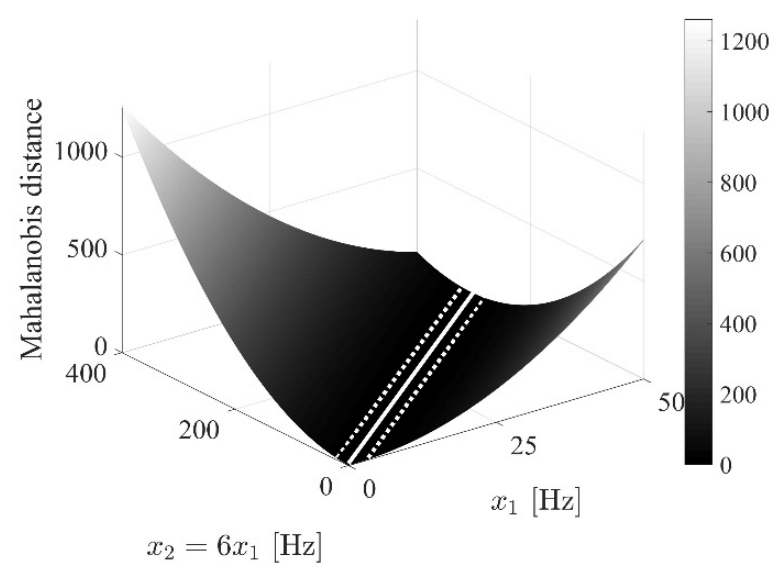

Fig. 6. Determination of the Mahalanobis distance thresholds.

Intuitively, the lowest Mahalanobis distance is located on the trend line itself, while a slightly higher Mahalanobis distance value is found for the lateral dashed lines which indicate the interval boundaries. Such value can be used to discriminate between an affiliated and a not affiliated frequency, and can be as well pre-calculated and stored together with $\boldsymbol{\mu}$ and $\boldsymbol{C}$.

\section{E. Trend line intervals overlap at low frequencies}

The presence of trend line intervals generates an interesting effect at low frequencies, shown in the example of Fig. 7 which refers to the case of $6^{\text {th }}$ and $12^{\text {th }}$ harmonic trend lines.

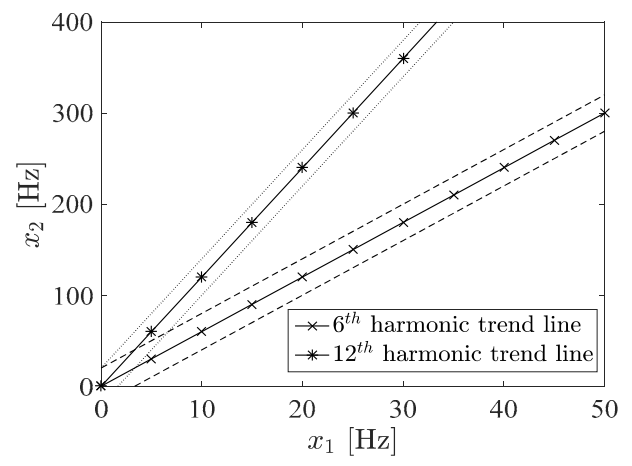

Fig. 7. Overlap of trend line intervals at low frequencies. 
In this example, fundamental frequencies approximately below $5 \mathrm{~Hz}$ fall into the interval of both trend lines, and therefore they could be a potential affiliation to both harmonics. This issue can be easily solved by selecting the trend line which is closest to the observed frequency, or in other words to choose the trend line that exhibits the lowest Mahalanobis distance to the observation. By relying on the repetition of the classification process over a certain number of FFTs, the likelihood of a correct interpretation of the result is greatly improved.

\section{EXPERIMENTAL RESULTS}

The proposed classification method is implemented and tested on the experimental setup shown in Fig. 8, composed by two electric machines in a back-to-back configuration. The machine under test is an $11-\mathrm{kW}$ synchronous reluctance machine (SynRM, on the right side of Fig. 8, see parameters in Table 1), while the load machine is an $11-\mathrm{kW}$ interior permanent magnet motor (IPM, on the left side of Fig. 1). Although the machine under test is a SynRM, the implemented algorithms are not tailored to it and operate with other motor types as well. A torque meter under the black protection cover is connected on the shaft. The IPM torque is regulated via an off-the-shelf 22-kW ABB ACS850 (upperright side of the picture). The SynRM prototype is connected to the power unit of an ABB ACS850, where the control board has been replaced by a custom interface and connected to the digital I/Os of an OPAL-RT Technologies OP5600 device (black box on the upper-left side of the figure). The OP5600 is equipped with a quad-core Intel DSP processor running at 2.4 $\mathrm{GHz}$ and a Virtex 6 FPGA. The phase currents and the DCbus voltage are measured with a custom measurement box (the grey box with a fan behind the load machine) also connected to the OP5600.

The control algorithm used to regulate the SynRM speed and torque is a conventional field-oriented control (FOC), with a switching frequency of $4 \mathrm{kHz}$.

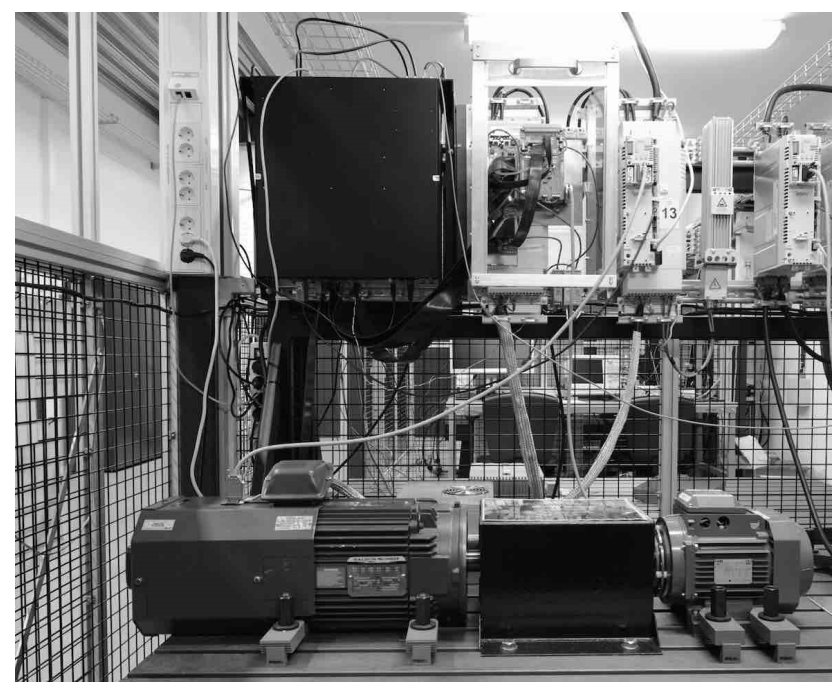

Fig. 8. Overview of the experimental setup.
TABLE 1. 11-KW SYNRM PARAMETERS.

\begin{tabular}{cc}
\hline Parameter name & Parameter value \\
\hline Nominal voltage & $400 \mathrm{~V}$ \\
Nominal current & $18 \mathrm{~A}$ \\
Nominal speed & $6000 \mathrm{rpm}$ \\
Nominal torque & $17 \mathrm{Nm}$ \\
Pole pairs & 2 \\
Stator resistance & $0.72 \Omega$ \\
$d$-axis inductance (magnetic linear region) & $80 \mathrm{mH}$ \\
$q$-axis inductance (magnetic linear region) & $60 \mathrm{mH}$ \\
Inertia (SynRM) & $0.00351 \mathrm{kgm}^{2}$ \\
Inertia (complete back-to-back connection) & $0.034 \mathrm{kgm}^{2}$ \\
\hline
\end{tabular}

\section{A. Experiment \# 1}

In this first test, the IGBT dead-time compensation was intentionally turned off, in order to introduce $6^{\text {th }}$ harmonics in the phase current waveforms and thus the current vector magnitude [3]. The phase currents and current vector magnitude of the machines are regulated at a frequency of 0.8 $\mathrm{Hz}$, as shown in Fig. 9. The classification algorithm was configured for finding the $5^{\text {th }}$ and $6^{\text {th }}$ harmonics of the current magnitude.

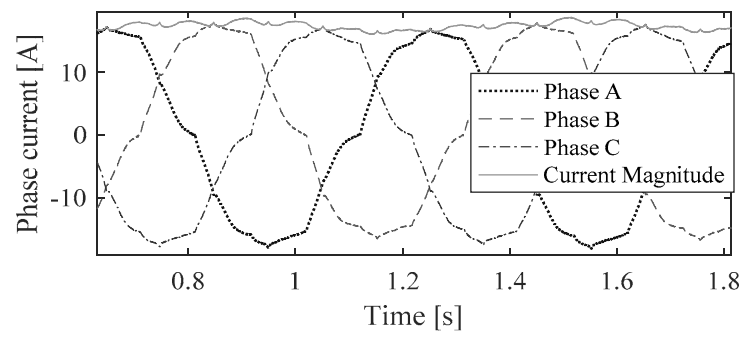

Fig. 9. Phase currents and current magnitude without IGBT dead-time compensation and visible $6^{\text {th }}$ harmonics.

The real-time FFT analysis on the current magnitude returned two non-negligible frequencies, which are marked with the black circles on harmonic representation in Fig. 10. The closeness of the circles indicates that some frequency resolution and spectral leakage issues were present, since two frequencies with non-negligible magnitude were found instead of simply one. However, as shown in Fig. 11, both points were falling into the Mahalanobis distance boundaries of the $6^{\text {th }}$ harmonic trend line. Therefore, the algorithm was able to correctly indicate the presence of a $6^{\text {th }}$ harmonic, cleaning the signal from frequency resolution, spectral leakage and noise issues.

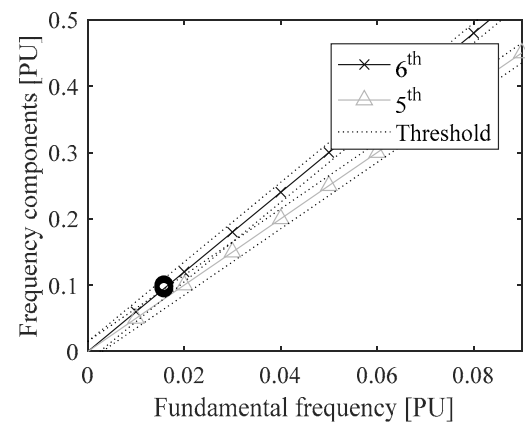

Fig. 10. Graphical representation of the $5^{\text {th }}$ and $6^{\text {th }}$ harmonic trends together with the frequencies found by the real-time FFT and marked with circles. 


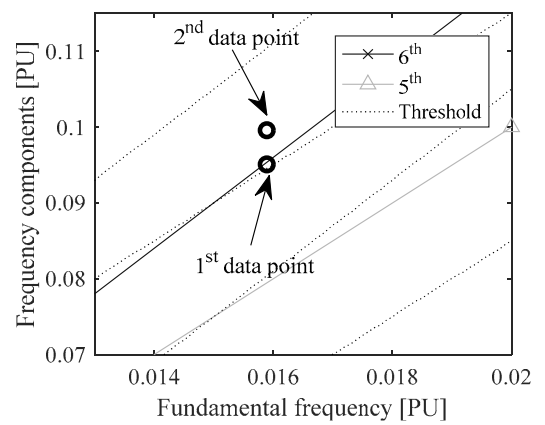

Fig. 11. Zoomed in view of the found frequencies by the real-time FFT.

\section{B. Experiment \# 2}

A similar test was performed where multiple frequencies were injected on purpose in the phase currents, covering the $1^{\text {st }}$, $2^{\text {nd }}, 3^{\text {rd }}$ and $6^{\text {th }}$ harmonics of the rotating speed. The electric frequency, the phase current magnitude and the torque of the drive under test are shown in Fig. 12. The FFT analysis of the current magnitude is shown in Fig. 13, together with the threshold for extracting the significant peaks (all the spectral lines with a magnitude above the given threshold are sent to the classification algorithm). It is noticeable that all harmonics but the $3^{\text {rd }}$ have a magnitude larger than the threshold, including the $\mathrm{DC}$ value. Lowering the threshold would have included the $3^{\text {rd }}$ harmonic as well. The threshold must therefore be intended as a customizable parameter.

The found frequencies (more than three due to spectral leakage, frequency resolution and noise) are represented in the harmonic diagram of Fig. 14 with circles. The classification algorithm based on the Mahalanobis distance was configured to detect the $1^{\text {st }}$ and the $6^{\text {th }}$ component of the electric frequency only, and it performed the task successfully since the boundaries around the trend lines were correctly set to avoid spectral leakage and frequency resolution issues. A zoom of the circles falling in the two trend lines is shown in Fig. 15 and Fig. 16. The group of circles falling into the same trend line were recognised as one harmonic, thus cleaning the measurements from unnecessary multiple entries. On the other hand, injected frequencies without any corresponding trend line were recognized as unknown ones, and returned as such for further analysis outside the drive control board.
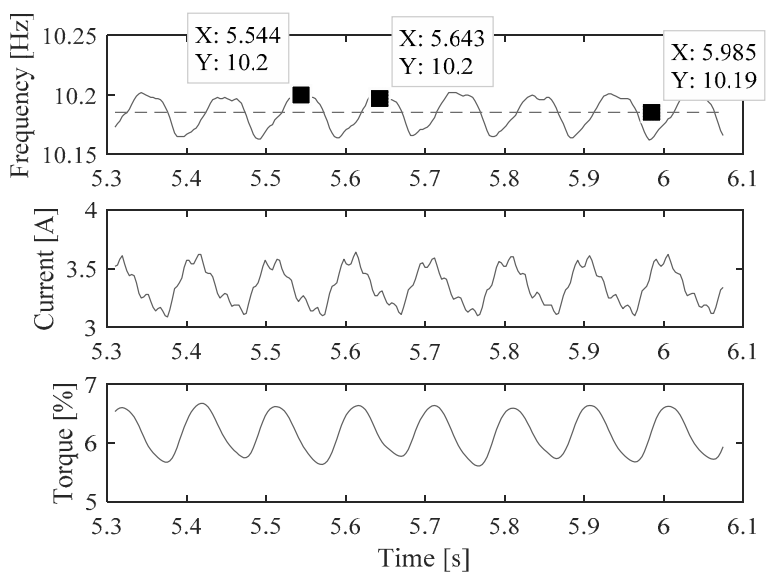

Fig. 12. Electric frequency, current magnitude and torque in the drive under test, with $1^{\text {st }}$ and $6^{\text {th }}$ harmonics and constant injected frequencies.

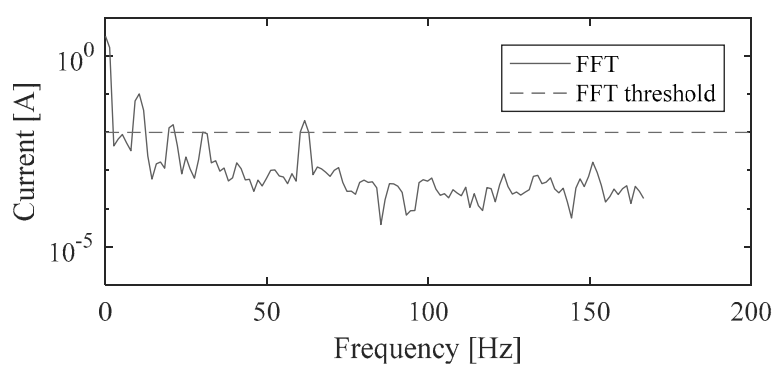

Fig. 13. FFT of the drive current magnitude.

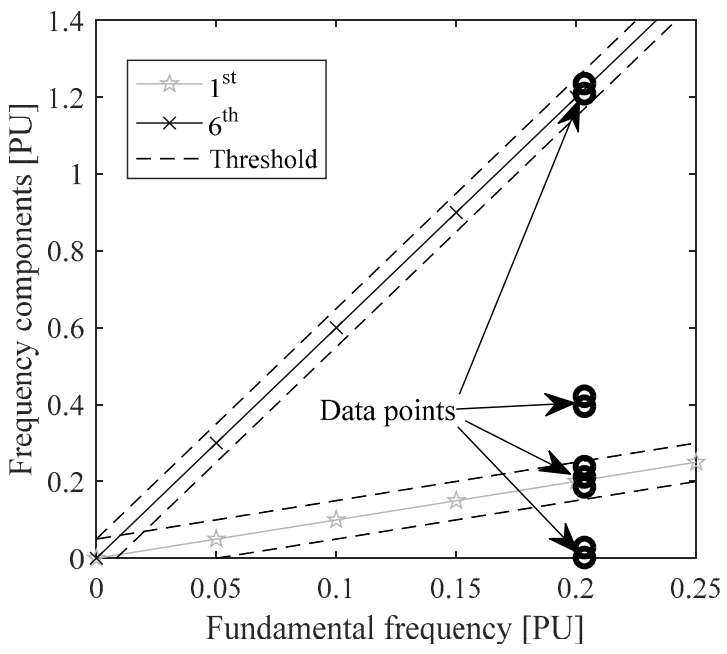

Fig. 14. Harmonic representation of the found frequencies in the test $\# 2$.

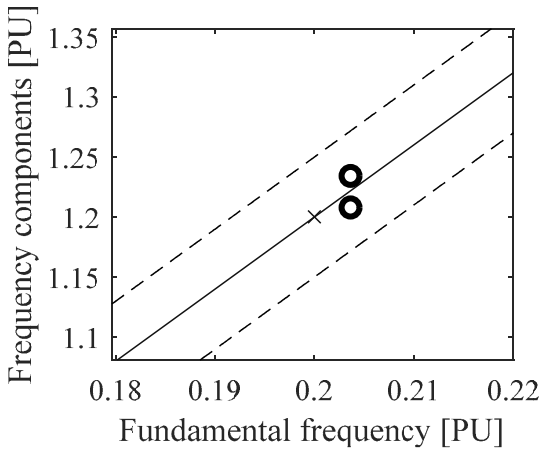

Fig. 15. Closer view of the circles in the $6^{\text {th }}$ harmonic trend line in the test $\# 2$.

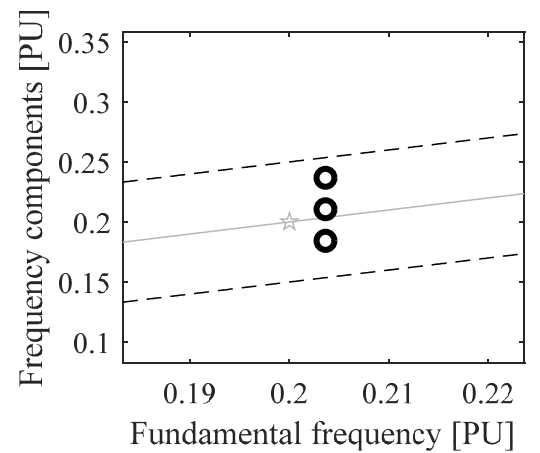

Fig. 16. Closer view of the circles in the $1^{\text {st }}$ harmonic trend line in the test $\# 2$. 


\section{CONCLUSIONS}

The mathematical concept of Mahalanobis distance is exploited in this paper to realise an algorithm which is capable of segregating known harmonics in a drive system, based on the results of a real-time frequency analysis. The theoretical background and the application to electric drives is explained in detail, together with a description of the main implementation details. The algorithm proves to be an interesting tool for monitoring and diagnostics, where an increased robustness of harmonic detection with respect to frequency resolution, spectral leakage and signal noise is found. Experimental results obtained on a laboratory setup demonstrate the validity of the concept, which can be extended to any motor type and any harmonic of interest.

\section{REFERENCES}

[1] J. Sopanen, V. Ruuskanen, J. Nerg, and J. Phyrhönen, "Dynamic torque analysis of a wind turbine drive train including a direct-driven permanent-magnet generator," IEEE Trans. Ind. Electron., vol. 58, no. 9, pp. 3859-3867, Sep. 2011

[2] W. L. Roux, R. G. Harley, and T. G. Habetler, "Detecting rotor faults in low power permanent magnet synchronous machines," IEEE Trans. Power Electron., vol. 22, no. 1, pp. 322-328, Jan. 2007.
[3] S. Bolognani, L. Peretti, and M. Zigliotto, "Repetitive-control-based self-commissioning procedure for inverter non-idealities compensation," IEEE Trans. Ind. Appl., vol. 44, no. 5, pp. 1587-1596, Sep./Oct. 2008.

[4] R. R. Obaid, T. G. Habetler, and R. M. Tallam, "Detecting load unbalance and shaft misalignment using stator current in inverter-driven induction motors," in Proceedings of the IEEE International Electric Machines and Drives Conference (IEMDC 2003), vol. 3, Madison, Wisconsin, USA, Jun. 1-4 2003, pp. 1454-1458.

[5] D. S. L. Dolan and P. W. Lehn, "Simulation model of wind turbine 3p torque oscillations due to wind shear and tower shadow," IEEE Trans. Energy Convers., vol. 21, no. 3, pp. 717-724, Sep. 2006.

[6] D.H. Lee, J.H. Lee, J.W. Ahn, "Mechanical vibration reduction control of two-mass permanent magnet synchronous motor using adaptive notch filter with fast Fourier transform analysis", IET Electr. Power Appl., 2012, Vol. 6, Iss. 7, pp 451-461.

[7] Haitham Hassanieh, Piotr Indyk, Dina Katabi, and Eric Price, "Simple and Practical Algorithm for Sparse Fourier Transform," in Symposium on Discrete Algorithms, Kyoto, 2012, pp. 1183-1194.

[8] C. Ma, Y. Kamp, and L. F. Willems, "Robust signal selection for linear prediction analysis of voiced speech," Speech Communication, no. 12, pp. 69-81, 1993.

[9] P.C. Mahalanobis, "On the generalized distance in statistics," Asiat. Soc. Bengal, vol. 2, no. 1, pp. 541-588, April 1936.

[10] F. J. Harris, "On the use of windows for harmonic analysis with the discrete Fourier transform", Proceedings of the IEEE, vol. 66, no. 1, pp. 51-83, Jan. 1978. 